\section{Tuberculose na cidade de Salvador, Bahia, Brasil: o perfil na década de 1990}

\author{
Tuberculosis in Salvador, Bahia, Brazil, in the 1990s
}

\begin{abstract}
The characteristics of tuberculosis (TB) cases and deaths were analyzed in order to characterize the epidemiological profile of TB (incidence and mortality) in Salvador, Bahia, Brazil, in the 1990s. Annual incidence and mortality rates were calculated by gender, age bracket, and clinical forms of the disease using databases from the Tuberculosis Information System of the Bahia State Health Secretariat and the Mortality Information System of the Brazilian Ministry of Health. TB spatial distribution was analyzed according to health district. Cases and deaths were predominantly in males in the 15 to 39 year group. The pulmonary form showed the highest incidence and mortality. The existing data did not corroborate the hypothesis that AIDS/TB coinfection might contribute to maintaining the high mortality rates. The greater occurrence of tuberculosis in certain health districts may be associated with population density and unfavorable living conditions.
\end{abstract}

Tuberculosis; Information Systems; Mortality; Incidence
Maria Izabel Mota Xavier 1

Maurício Lima Barreto 2

\section{Introdução}

Em anos recentes, a tuberculose tornou-se uma doença reemergente nos países desenvolvidos, além de ter mantido a ocorrência em níveis elevados e eventualmente crescentes nos países em desenvolvimento. Dentre os fatores relacionados com este fato, destacam-se os fatores sociais como determinante principal associado à ocorrência da doença. Além disso, a insuficiência de recursos para o cuidado da saúde e a falta de decisão política por parte dos gestores para priorizar as ações efetivas de controle têm colaborado para agravar o quadro. A situação veio agravarse com o advento da epidemia da AIDS, quando houve um aumento de $12 \%$ no número de casos da tuberculose nos países desenvolvidos 1 . A coinfecção, associada ao abandono do tratamento pelo doente, têm contribuído para o crescimento da proporção de casos da doença causados por cepas multidroga-resistentes, o que acarreta efeitos ainda mais devastadores. Essas cepas se transmitem para novos pacientes, ocasionando formas mais severas e com elevado custo econômico e social.

Diante do número elevado de casos de tuberculose e da alta mortalidade no mundo, em 1993, a Organização Mundial da Saúde (OMS), declarou estado de emergência global. Destacase ainda que $1 / 3$ da população mundial encontra-se infectada pelo Mycobacterium tuberculosis 1 . Estimativas apontaram para a existência de 
1,86 bilhão de pessoas infectadas no mundo e a ocorrência de mais de 1,8 milhão de óbitos por ano ${ }^{2}$.

Os países em desenvolvimento apresentam as mais altas taxas de incidência da doença. No caso do Brasil, os números são considerados preocupantes. Em 1999, a taxa de incidência registrada foi acima de 50/100 mil habitantes, estando o país em décimo lugar em número total de casos. Nesse mesmo período, o Estado da Bahia apresentou taxa de incidência de 60/100 mil habitantes, contribuindo com cerca de $10 \%$ dos casos do país, posicionando-se em terceiro lugar em número de casos, quando comparado com os demais estados. A Bahia tem ainda registrado, a cada ano, uma média de 400 óbitos, como conseqüência da tuberculose (Secretaria de Saúde do Estado da Bahia - SESAB; dados não publicados).

Nas capitais, a doença apresenta as mais altas incidências devido à alta densidade demográfica, aos bolsões de pobreza, ao elevado risco de infecção. Deve-se também considerar que nessas cidades devido à concentração de maior oferta de serviços de saúde, existem melhores condições de diagnóstico e tratamento. No Município de Salvador, as taxas de incidência registradas ultrapassam de 100/100 mil habitantes ao ano, apesar das reduções observadas nas últimas décadas.

Este estudo tem o objetivo de traçar o perfil epidemiológico da tuberculose, identificando as populações de maior risco e as características dos casos da doença, no Município de Salvador, na década de 1990.

\section{Metodologia}

Este estudo foi realizado no Município de Salvador, capital do Estado da Bahia, que apresentou no ano de 2000 , população de 2.331 .612 de habitantes, 50,3\% dos quais do sexo masculino. Sua taxa de urbanização foi de $99,9 \%$, possui uma área de extensão territorial de $709,5 \mathrm{~km}^{2}$ e densidade demográfica de 3.286,27 habitantes $/ \mathrm{km}^{2} 3$.

Como fonte de informação para os casos de tuberculose utilizou-se o banco de dados do Sistema de Informações de Tuberculose da SESAB, que registra os casos da doença detectados pela vigilância epidemiológica. Foram incluídos neste estudo os casos novos notificados entre os residentes do Município de Salvador, no período de 1990 a 2000. Definiu-se como "caso novo" doente com tuberculose que nunca usou ou usou por menos de um mês drogas antituberculosas, tendo sido o diagnóstico realizado utilizando-se os métodos laboratorial ou radiológico, ou ainda, baseado em critério clínico 4 .
Os dados sobre mortalidade no período foram obtidos do Sistema Informação de Mortalidade do Ministério da Saúde, que tem dados definitivos relativos à Bahia do período de 1990 a 1998. Para o ano de 1999, utilizou-se as informações do banco de dados sobre mortalidade da SESAB.

Para traçar o perfil epidemiológico da tuberculose, foram considerados o número global de óbitos e o número de casos da doença, ocorridos na década de 90. Foi analisada a freqüência da distribuição das variáveis de maior interesse disponível no banco de dados, como: sexo, faixa etária, forma clínica, método diagnóstico utilizado, a existência da co-infecção AIDS/tuberculose e ocorrência de internamento. Observou-se também a distribuição espacial da tuberculose nos Distritos Sanitários que compõem o Município de Salvador.

Para possibilitar a identificação do comportamento da doença na década analisada, foram calculadas taxas médias de mortalidade e de incidência para o período de 1990 a 2000. Nesse cálculo, o numerador correspondeu ao total de casos de tuberculose notificados no período de 1990 a 2000, e o número de óbitos por essa doença no período de 1990 a 1999 entre os residentes do Município de Salvador. Para o denominador, considerou-se a soma da população de Salvador dos anos censitários de 1991 e 2000, a contagem da população de 1996 e estimativa da SESAB para os demais anos. Utilizou-se o programa Epi Info 5.0 (Centers for Disease Control and Prevention, Atlanta, Estados Unidos) para estruturar o banco de dados e proceder as análises.

\section{Resultados}

Registrou-se no Município de Salvador, entre 1990 a 2000, 31.903 casos novos de tuberculose por todas as formas, significando taxa média anual de incidência de 131,5/100 mil habitantes. As taxas de incidência mais elevadas foram encontradas no grupo de 15 a 39 anos (224/100 mil habitantes) e nos maiores de 60 anos (183,1/100 mil habitantes). Houve predomínio do sexo masculino $(60,1 \%)$. A faixa etária com maior proporção de casos foi a de 15 a 39 anos $(60,4 \%)$, seguida da faixa de 40 a 59 anos (24,1\%) (Tabela 1).

A forma pulmonar da doença contribuiu com $85,3 \%$ dos óbitos. Das formas extrapulmonares, a forma miliar correspondeu a 17,5\% dos óbitos e a meningite tuberculosa representou $14,6 \%$, ficando os demais casos como de outras localizações (67,9\%) (Tabela 2).

Em relação à mortalidade no período de 1990 a 1999, ocorreram 1.859 óbitos por tuberculose 
Tabela 1

Número de óbitos e casos, percentual e taxa média de incidência da tuberculose segundo características demográficas Salvador, Bahia, Brasil, 1990-2000.

\begin{tabular}{|c|c|c|c|c|c|c|c|}
\hline \multirow[b]{2}{*}{ Característica } & \multicolumn{3}{|c|}{ Casos } & \multicolumn{4}{|c|}{ Óbitos } \\
\hline & $n$ & $\%$ & $\begin{array}{c}\text { Taxa } \\
\text { (por 100.000) }\end{array}$ & $n$ & $\%$ & $\begin{array}{c}\text { Taxa } \\
\text { (por 100.000) }\end{array}$ & $\begin{array}{l}\text { Letalidade } \\
\text { (\%) }\end{array}$ \\
\hline \multicolumn{8}{|l|}{ Sexo * } \\
\hline Masculino & 19.172 & 60,1 & 161,2 & 1.287 & 69,5 & 11,6 & 6,5 \\
\hline Feminino & 12.731 & 39,9 & 99,3 & 564 & 30,4 & 5,3 & 4,8 \\
\hline \multicolumn{8}{|l|}{ Faixa-etária (anos) } \\
\hline $0-4$ & 927 & 2,9 & 39,9 & 39 & 2,1 & 1,8 & 4,2 \\
\hline $5-14$ & 1.425 & 4,5 & 26,6 & 23 & 1,2 & 0,5 & 1,6 \\
\hline $15-39$ & 19.278 & 60,4 & 224,0 & 589 & 31,7 & 7,6 & 3,1 \\
\hline $40-59$ & 7.687 & 24,1 & 105,7 & 726 & 39,1 & 10,5 & 9,4 \\
\hline $60 e+$ & 2.549 & 8,0 & 183,1 & 432 & 23,2 & 34,6 & 16,9 \\
\hline Idade incorreta & 37 & 0,1 & - & 50 & 2,7 & - & - \\
\hline Total & 31.903 & 100,0 & 131,5 & 1.859 & 100,0 & 8,5 & 5,8 \\
\hline
\end{tabular}

Fonte: Diretoria de Informação e Comunicação em Saúde, Secretaria de Saúde do Estado da Bahia.

* Em oito óbitos não havia definição do sexo.

Tabela 2

Número de casos e óbitos, proporção e taxa média de incidência de tuberculose conforme a forma clínica. Salvador, Bahia, Brasil, 1990-2000.

\begin{tabular}{|c|c|c|c|c|c|c|}
\hline \multirow[b]{2}{*}{ Formas } & \multicolumn{3}{|c|}{ Casos } & \multicolumn{3}{|c|}{ Óbitos } \\
\hline & $\mathrm{n}$ & $\%$ & $\begin{array}{c}\text { Taxa média } \\
\text { (por 100.000) }\end{array}$ & $\mathrm{n}$ & $\%$ & $\begin{array}{c}\text { Taxa média } \\
\text { (por 100.000) }\end{array}$ \\
\hline Pulmonar & 27.216 & 85,3 & 112,2 & 1.585 & 85,3 & 7,2 \\
\hline Positiva & 17.868 & 65,7 & 73,7 & - & - & - \\
\hline Negativa & 5.142 & 18,9 & 21,2 & - & - & - \\
\hline \multicolumn{7}{|l|}{ Sem confirmação } \\
\hline Baciloscópica & 4.131 & 15,2 & 17,0 & - & - & - \\
\hline Extrapulmonar & 4.687 & 14,7 & 19,3 & 274 & 14,7 & 1,2 \\
\hline Pleural & 2.502 & 53,4 & 10,3 & - & - & - \\
\hline Ganglionar periférica & 943 & 20,1 & 3,9 & - & - & - \\
\hline Genito-urinária & 119 & 2,5 & 0,5 & - & - & - \\
\hline Ocular & 39 & 0,8 & 0,2 & - & - & - \\
\hline Miliar & 162 & 3,5 & 0,7 & 48 & 17,5 & 0,2 \\
\hline Óssea & 137 & 2,9 & 0,6 & - & - & - \\
\hline Meningite & 168 & 3,6 & 0,7 & 40 & 14,6 & 0,2 \\
\hline Outras & 617 & 13,2 & 2,5 & 186 & 67,9 & 0,8 \\
\hline Total & 31.903 & 100,0 & 131,5 & 1.859 & 100,0 & 8,5 \\
\hline
\end{tabular}

Fonte: Diretoria de Informação e Comunicação em Saúde, Secretaria de Saúde do Estado da Bahia. 
no Município de Salvador, correspondendo à taxa média anual de 8,5/100 mil habitantes. A taxa média de mortalidade mais elevada foi encontrada entre os maiores de 60 anos (34,6/100 mil habitantes). O sexo masculino contribuiu com $69,5 \%$ dos óbitos. O maior número de óbitos ocorreu na faixa de 40 a 59 anos $(39,1 \%)$, seguida da de 15 a 39 anos (31,8\%). A letalidade geral foi de $5,8 \%$, atingindo níveis mais altos no sexo masculino e na população acima de 40 anos de idade (Tabela 1).

A forma pulmonar foi a mais freqüente em meio aos casos de tuberculose ocorridos em Salvador $(85,3 \%)$. Dentre as ocorrências de tuberculose da forma pulmonar, com baciloscopia positiva, representou $65,7 \%$ dos casos; entre as formas extrapulmonares, registrou-se com maior freqüência a forma pleural (53,4\%), seguido da forma ganglionar com $20,1 \%$ dos casos; as formas miliares e a meningite tuberculosa contribuíram com $3,5 \%$ e $3,6 \%$ dos casos extrapulmonares, respectivamente. As taxas médias de incidência encontradas foram 112,2/100 mil habitantes e 19,3/100 mil habitantes, para as formas pulmonares e extrapulmonares, respectivamente (Tabela 2).

Com relação aos procedimentos utilizados para o diagnóstico da tuberculose, constatou-se que, além da baciloscopia realizada em $72,1 \%$ dos casos, a radiografia foi realizada em $62,4 \%$ dos doentes, apresentando resultado suspeito em $93,2 \%$. A cultura foi realizada em $17,3 \%$ dos pacientes e o resultado positivo foi confirmado em 9,3\% deles; em 85,6\% desses, o resultado não foi registrado no momento da notificação, por estar o exame em andamento. Possivelmente, significando que devido ao tempo para o seu resultado e a difícil acessibilidade, ela termina sendo desprezada como recurso diagnóstico. Situação semelhante foi encontrada no teste de sorologia para HIV, em que $28,8 \%$ dos testados apresentaram resultado positivo, e em $46,9 \%$ o exame encontrava-se em processo, no ato da notificação. Do total de casos, apenas 6,6\% realizaram esse teste (Tabela 3).

Em relação à associação com a AIDS, verificou-se que 394 casos de tuberculose ocorreram em pacientes com a AIDS. Comparando-se com os casos que não referiram a AIDS, observou-se que as formas extrapulmonares ocorreram em $25,6 \%$ dos casos com AIDS e em 14,3\% dos casos sem AIDS $\left(\chi^{2}=36,2 ; p<0,01\right)$ (Tabela 4$)$.

Na distribuição espacial da tuberculose pelos distritos sanitários da cidade de Salvador, foram verificadas as mais elevadas taxas de incidência (por 100 mil habitantes), nos distritos do Centro Histórico $(189,4)$, Liberdade $(158,8)$, Itapagipe, São Caetano/Valéria $(148,1)$ e Subúrbio Ferroviário $(143,4)$ (Figura 1)
Tabela 3

Número e proporção de casos de tuberculose, segundo método diagnóstico utilizado. Salvador, Bahia, Brasil, 1990-2000

\begin{tabular}{|c|c|c|}
\hline Exames & n & $\%$ \\
\hline \multicolumn{3}{|l|}{ Baciloscopia } \\
\hline Realizada & 23.010 & 72,1 \\
\hline Positiva & 17.868 & 77,7 \\
\hline Negativa & 5.142 & 22,3 \\
\hline Não realizada & 4.131 & 15,2 \\
\hline Sem informação & 4.582 & 12,7 \\
\hline \multicolumn{3}{|l|}{ Radiografia } \\
\hline Realizada & 19.919 & 62,4 \\
\hline Suspeito & 18.569 & 93,2 \\
\hline Normal & 830 & 4,2 \\
\hline Outras alterações & 520 & 2,6 \\
\hline Não realizada & 932 & 2,9 \\
\hline Sem informação & 11.052 & 34,7 \\
\hline \multicolumn{3}{|l|}{ Cultura } \\
\hline Realizada & 5.509 & 17,3 \\
\hline Positiva & 510 & 9,3 \\
\hline Negativa & 281 & 5,1 \\
\hline Em andamento & 4.718 & 85,6 \\
\hline Não realizada & 15.302 & 48,0 \\
\hline Sem informação & 11.092 & 34,7 \\
\hline \multicolumn{3}{|l|}{ Sorologia para HIV } \\
\hline Realizada & 2.105 & 6,6 \\
\hline Positivo & 608 & 28,9 \\
\hline Negativo & 509 & 24,2 \\
\hline Em andamento & 988 & 46,9 \\
\hline Não realizada & 18.657 & 58,5 \\
\hline Sem informação & 11.141 & 34,9 \\
\hline
\end{tabular}

Fonte: Diretoria de Informação e Comunicação em Saúde, Secretaria de Saúde do Estado da Bahia.

Tabela 4

Número de casos de tuberculose associado à AIDS conforme a forma clínica da doença. Salvador, Bahia, Brasil, 1990-2000.

\begin{tabular}{lccc}
\hline AIDS & $\mathbf{n}$ & Pulmonar & Extrapulmonar \\
\hline Sim & 394 & 74,4 & 25,6 \\
Não & 4.639 & 85,7 & 14,3 \\
\hline
\end{tabular}

Fonte: Diretoria de Informação e Comunicação em Saúde, Secretaria de Saúde do Estado da Bahia. $p=0,01$. 


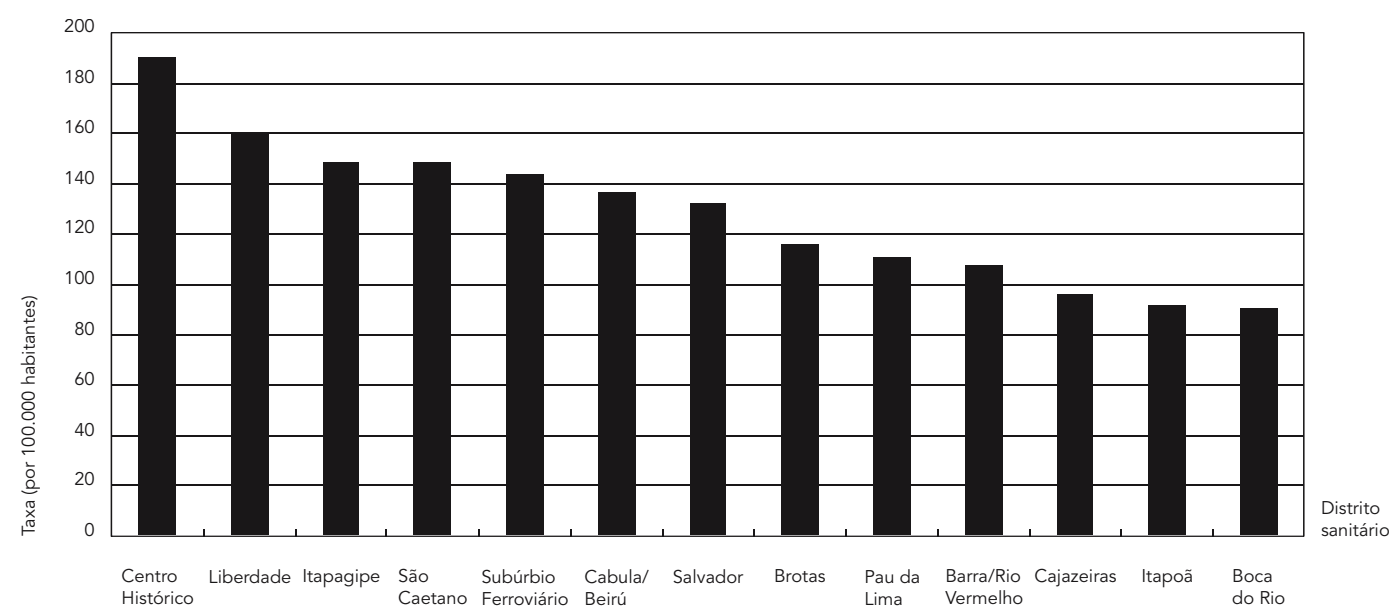

\section{Discussão}

O total de óbitos por tuberculose, registrados no Município de Salvador, no período de 1990 a 1999, correspondeu à metade do total de óbitos ocorridos no Estado da Bahia, apesar da capital concentrar apenas 18,6 \% da população do Estado. A taxa média de mortalidade no período foi superior à encontrada no estado, sendo maior que o dobro da registrada no país, o que leva a supor que alguns fatores podem contribuir para manutenção dessas taxas em níveis elevados ou, ainda, as ações de controle, desencadeadas nesse período, não têm sido suficientes para a redução da mortalidade por tuberculose nesse município, uma vez que, o diagnóstico tardio e tratamento inadequado corroboram para o aumento da mesma. Em outros locais, têm sido apresentadas evidências de que a manutenção das taxas elevadas da mortalidade esteja associada à ocorrência da co-infecção HIV/tuberculose. Entretanto, em Salvador, os dados existentes enquanto não permitam uma análise mais detalhada dessa associação, não sugerem uma importância significativa da infecção pelo HIV no padrão da tuberculose na cidade do Salvador. No Município de São Paulo, no período de 1985 a 1995, a elevação observada das taxas de mortalidade por tuberculose foi associada por Antunes \& Waldman 5 à ocorrência da AIDS. Entre os maiores de 60 anos, a taxa média de mortalidade foi a mais elevada, podendo estar associada não só à ocorrência de desordens crônicas no idoso, mas também às dificuldades de diagnóstico nessa faixa etária, que retarda o início do tratamento, aumentando a mortalidade 6,7 .

Ressalta-se que a proporção de óbitos por tuberculose da forma pulmonar (85,3\%) é igual à proporção de casos incidentes desta forma (Tabela 2). No Município de São Paulo, a forma pulmonar contribuiu com $73,8 \%$ dos óbitos ${ }^{8}$. No entanto, é possível que o retardo no diagnóstico, tratamentos inadequados além da baixa imunidade estejam contribuindo para a alta mortalidade, mesmo nos casos da forma pulmonar.

No início da década de 90, foi constatado, em Salvador, um aumento do número de casos de tuberculose, atingindo seu ápice em 1994. A incidência notificada da tuberculose também foi elevada no período. Comparando-se Salvador com outras capitais do país de alta incidência, seus níveis aproximaram-se daqueles do Município do Rio de Janeiro, que apresentou, no período de 1995 a 1997, taxa média de incidência de 120/100 mil habitantes 9, e estiveram acima do Município de São Paulo, que registrou taxa média de 58,6/ 100 mil habitantes entre 1990 e 1997 (Divisão de Tuberculose, Centro de Vigilância Epidemiológica de São Paulo; dados não publicados).

A menor ocorrência da doença, nos menores de 14 anos, pode ser justificada pela dificuldade em diagnosticar os casos nesta faixa etária, já 
que, trata-se de forma menos severa da doença, o que leva à realização de testes terapêuticos sem confirmação diagnóstica e provavelmente não são notificados após resposta terapêutica favorável ou pelo aumento das coberturas vacinais no Município de Salvador, que elevaram-se a partir de 1994 e mantiveram-se em 100\% após o ano de 1997. A proteção conferida pela vacina BCG varia de $0 \%$ a $80 \%$, contra a forma pulmonar, e de $46 \%$ a $100 \%$ contra as formas graves da doença na criança 10. Estudo recente em Salvador mostrou uma efetividade da ordem de $27 \%$ contra a forma pulmonar da doença e de $37 \%$ contra a forma extrapulmonar 11. Em relação à meningite tuberculosa, estudo também em Salvador mostrou nível de proteção da ordem de $99,5 \% 12$, similar ao encontrado em outras partes do mundo.

A maior proporção dos casos de tuberculose em Salvador ocorreu na faixa de 15 a 39 anos, portanto semelhante à distribuição encontrada no país. Também nessa faixa, foi registrada a mais alta taxa de incidência da doença. Em algumas áreas este achado tem sido associado à alta prevalência do HIV e ao alcoolismo 7,13. Os maiores de 60 anos também apresentaram incidência elevada. Nessa faixa a associação está relacionada à alta proporção de indivíduos com infecção latente de tuberculose 7 . Nos países desenvolvidos, com um controle adequado da doença, tem havido mudança na característica epidemiológica da tuberculose, com aumento no grupo de idosos. Essa mudança está associada ao crescimento da população idosa como conseqüência do aumento da expectativa de vida. Entre os idosos, pela freqüência das desordens crônicas, ocorre reativação da infecção endógena, além da dificuldade de diagnóstico, que retarda o início do tratamento e aumenta a mortalidade. No Brasil, acrescenta-se a esses fatores as condições precárias de moradia e o difícil acesso ao sistema de saúde, como também as desvantagens vividas pelo idoso na esfera econômica e social 7. Em outros estudos, realizados no Brasil 7, em Havana, Cuba ${ }^{14}$, e na Inglaterra ${ }^{6}$, constatou-se aumento nas taxas da tuberculose na população idosa, evidenciando uma mudança no perfil da doença.

Entre as formas pulmonares da tuberculose, a maioria $(65,7 \%)$ teve o resultado da baciloscopia positiva. Essa forma da doença, por apresentar maior infectividade, é a de maior interesse para as atividades de controle de saúde pública. Por isso a importância do diagnóstico baseado no exame de baciloscopia do escarro, para a confirmação e o controle adequado dos casos, de modo a obter a interrupção da cadeia de transmissão da doença. Constatou-se que, entre aqueles acometidos pela forma pulmonar da tuberculose, 15,5\% não foram submetidos a esse procedimento. Outro fato que chama a atenção é a utilização freqüente da radiologia como método para o diagnóstico da tuberculose. Segundo normatização do Programa de Controle da Tuberculose, o uso da radiografia é restrito para algumas situações, como no caso de resultado de baciloscopia negativa, no diagnóstico em menores de 12 anos e nas formas extrapulmonares da doença 4 . Não é recomendado o diagnóstico da tuberculose pulmonar baseado somente em achados da radiografia, pois poderá levar a uma alta taxa de erros, decorrente da baixa especificidade deste método 15 . Natal \& Gerhardt Filho 16 demonstraram que 51,3\% dos pacientes teriam sido tratados desnecessariamente, caso o diagnóstico fosse baseado somente na radiografia.

Com relação à co-infecção AIDS/tuberculose, estimativas estabelecidas para o Brasil apontam para a existência de 500 mil pessoas infectadas pelo vírus HIV, sendo $17 \%$ destes casos detectados em pessoas acometidas pela tuberculose 17,18. No Município de Salvador, há registro de somente $1 \%$ dos casos com a co-infecção AIDS/ tuberculose no Sistema de Informações de Tuberculose da SESAB, podendo refletir uma subregistro devido a questões diagnósticas. Provavelmente, essa informação não está sendo obtida no momento do diagnóstico da tuberculose, já que, na rotina, a solicitação da sorologia para o teste de HIV ainda é muito baixa, apesar da orientação de que, na investigação clínica dos casos da tuberculose a sorologia deva ser considerada para o diagnóstico diferencial de todas as situações do paciente com HIV/AIDS 19. A Coordenação Nacional de Pneumologia Sanitária do Ministério da Saúde também recomenda investigar o HIV nos pacientes com diagnóstico de tuberculose, porém na prática isto não vem ocorrendo, conforme pode-se constatar, já que somente em uma pequena proporção de casos foi realizado o teste de sorologia para HIV, e em metade destes o resultado dos exames encontrava-se em andamento no momento da notificação, ficando sem registro no sistema de informações.

A maior ocorrência de casos de tuberculose extrapulmonar foi observada nos que referiram associação com a AIDS, diferentemente dos casos sem AIDS, confirmando que as formas extrapulmonares estão associadas a situações de fragilidade do sistema imunológico como ocorrem nos casos de AIDS (Tabela 4). Outros estudos corroboram para ratificar o resultado; no Rio de Janeiro, encontrou-se associação entre a tuberculose e a AIDS em torno de $29,3 \%$ dos casos, sendo $40,4 \%$ das formas clínicas extrapulmonares e/ou disseminadas 20. Kerr-Pontes et al. 21, 
no Estado do Ceará, constataram a presença da tuberculose em 19,3\% dos casos diagnosticados com AIDS, detectaram também a ocorrência significativamente maior de casos da forma extrapulmonar, com índices de $18,3 \%$ a $70 \%$. Dupon et al. 13 referem a ocorrência de $40 \%$ da forma extrapulmonar nos casos de AIDS em Aquitaine, Reino Unido 13.

Dentre os casos de meningite tuberculosa registrados em Salvador, menos de um terço foi admitido em unidade hospitalar. Tratando-se de uma das formas graves da doença, o internamento é fundamental para a realização do diagnóstico e da terapia adequada; o baixo percentual desse procedimento deve refletir falha do sistema de informações. Os casos de meningite podem ser tratados em qualquer unidade hospitalar, embora, em Salvador, o hospital de referência seja o Hospital Couto Maia, para onde são referendados a maioria dos casos da doença com essa forma.

A distribuição espacial da tuberculose em Salvador na década de 90 concentrou 55,9\% dos casos em cinco distritos sanitários, sendo as maiores taxas registradas nos distritos do Centro Histórico, Liberdade, Itapagipe e São Caetano. Esses distritos possuem elevada concentração populacional e, juntamente com o Subúrbio Ferroviário, representam mais da metade da população do município, sendo a população média nessas áreas superior a 250 mil habitantes, com exceção do distrito da Liberdade que, no ano de 2000, registrou população superior a 170 mil habitantes. A maior ou menor ocorrência de casos de tuberculose nos distritos sanitários pode ter associação com a concentração populacional ou com as condições de vida neles presentes, já que fatores sociais possuem alta relevância para a ocorrência dessa doença. Os distritos sanitários onde houve predominância do número de casos referem-se a áreas populosas e localizam-se na periferia do Município de Salvador. Outros estudos verificaram uma maior ocorrência de casos em residentes nos municípios com mais de 250 mil habitantes 14,22.

No entanto, o distrito Barra/Rio Vermelho, considerado como uma das áreas com melhores condições sócio-econômicas, também contribuiu com uma parcela importante no registro de casos, nesse período. Deve-se, entretanto, ressaltar que nesse distrito também estão presentes extensos bolsões de pobreza. Situação semelhante foi encontrada por Mangtani et al. 23 ao investigar a associação entre as privações sócio-econômicas e a alta prevalência de tuberculose em Londres; Carneiro \& Mota 24, espacializando a ocorrência da tuberculose de acordo com 67 zonas de informação de Salvador, em 1980, observaram correlação positiva entre a doença e a densidade populacional.

Considerando que, na década analisada, as taxas de incidência e de mortalidade por tuberculose foram elevadas no país, propostas para implementar ações foram desencadeadas, no plano nacional, com o objetivo de controlar o agravo. No ano de 1994, foi lançado pelo Ministério da Saúde o Plano Emergencial que priorizava o controle da tuberculose em 240 municípios, sendo um dos critérios de inclusão as elevadas taxas de incidência da doença. Nesse sentido, o Município de Salvador foi considerado prioritário e, portanto, garantiria a implementação dessas ações, acordadas entre o Ministério da Saúde, o município e o estado, através das Secretarias de Saúde. Em 1999, o Ministério da Saúde lançou o Plano Nacional de Controle da Tuberculose; por esse plano um bônus para financiar as ações passou a ser recebido pelo município mediante a realização do diagnóstico, tratamento e cura do doente de tuberculose 25 .

Convém salientar que, apesar da recomendação e da priorização das ações de controle no plano nacional, nem sempre as mesmas estão de acordo com as prioridades do Estado ou município, levando às vezes a um descompasso dessas ações. Apesar das estratégias de ação implementadas, e das ações de diagnóstico e tratamento mantidas, as taxas de incidência e de mortalidade permaneceram elevadas. Também nessa década, Salvador passou pelo processo de descentralização das ações de saúde, o que levou à gestão direta do Programa de Controle da Tuberculose pelo governo municipal. Entretanto, a esperada descentralização das ações para a atenção básica ocorre de maneira incipiente tendo em vista a baixa cobertura das estratégias do Programa Saúde da Família, o que tem dificultado a expansão do tratamento supervisionado, como medida para o acompanhamento adequado do tratamento, visando ao aumento do número de pacientes curados, pelo aumento da adesão ao tratamento e conseqüentemente reduzir multidroga resistência, a mortalidade, o sofrimento e o ônus social e político do país.

As coortes realizadas periodicamente pelo programa têm apontado melhora nos indicadores de avaliação em Salvador. Constatou-se aumento do percentual de cura para $81 \%$ nos anos de 1996 e 1997; entretanto, este percentual veio decrescendo até atingir $64,6 \%$ no ano 2000 . Segundo a OMS, para que haja redução do risco de infecção, o percentual de cura deve ser superior a $85 \%$, do contrário permanecem as fontes de contágio e aumenta a possibilidade de multidroga resistência pelo elevado percentual de abandono 26 . Em Salvador, esse índice atingiu $23,4 \%$ em 
1994, apresentou redução gradativa até alcançar 7,6\% no ano 2000; no entanto, essa análise ficou prejudicada, tendo em vista que, de um elevado percentual (23\%) dos casos não se tem informação da situação ao final do tratamento, o que demonstra a falta de acompanhamento adequado do doente pela equipe de saúde da unidade ou, ainda, o fato de o fluxo de informação existente não estar sendo capaz de responder às necessidades do programa.

\section{Conclusão}

Apesar das falhas existentes no Sistema de Informações de Tuberculose da SESAB, que levaram a algumas limitações no estudo, o que reforça o valor do registro e a vigilância da doença para identificar a população de risco e característica dos casos. O estudo concluiu que, as taxas médias de incidência e da mortalidade no Município de Salvador são elevadas. Alguns fatores podem contribuir para a manutenção do quadro, dentre eles, o uso inadequado das tecnologias disponíveis, como o diagnóstico tardio por não haver busca ativa de casos novos entre os sintomáticos respiratórios e os contactantes; a baixa cobertura do Programa que dificulta o acompanhamento apropriado dos casos em tratamento; a utilização inadequada do sistema de informação que compromete a qualidade dos dados, o que impossibilita a análise da incidência real e o resultado dos tratamentos para subsidiar a tomada de decisão com vistas a uma intervenção apropriada. Portanto, os recursos existentes para o controle da doença vêm sendo utilizados de forma inadequada e não têm assegurado o controle da doença, pois pouca alteração é observada na situação.
O maior risco da doença encontra-se entre os indivíduos do sexo masculino do grupo de adultos jovens, seguido pela população idosa, o que implica adoção de medidas de controle apropriadas para impedir a transmissibilidade da doença, como o diagnóstico precoce e a imediata instalação de tratamento, já que no grupo de idosos a forma cavitária é mais freqüente, acarretando aumento da transmissão da enfermidade para comunidade.

As informações sobre a co-infecção AIDS/ tuberculose são insuficientes para que seja mensurado o impacto da AIDS na incidência de tuberculose. Serão necessárias a realização de investigação adequada para se conhecer o diagnóstico da situação, e a articulação entre os dois programas (AIDS e tuberculose) para medir acuradamente a extensão da sobreposição dos dois agravos.

Apesar das propostas para implementar ações no plano nacional, estas não vêm sendo priorizadas no plano municipal, mesmo sendo acordadas entre a SESAB e o Ministério da Saúde, e com garantia do repasse de verbas para financiamento destas ações.

Para um controle eficaz da doença, faz-se necessária a sustentação de atividades de prevenção e controle. Em particular, é preciso prover assistência à saúde, identificar todo caso de tuberculose e referir para as unidades de saúde, assegurando o tratamento com sucesso. Essas ações contribuirão para a redução do risco de infecção pela eliminação das fontes de contágio, no entanto, essas medidas necessitam de ações articuladas com setores sociais, uma vez que a melhoria das condições de vida é essencial para um controle efetivo da tuberculose.

\section{Resumo}

Com o objetivo de caracterizar o perfil epidemiológico da tuberculose (incidência e mortalidade) no Município de Salvador, Bahia, Brasil, na década de 1990, foram analisadas as características demográficas, clínicas e laboratoriais dos casos e óbitos por esta doença ocorridos no período. Foram calculadas as taxas médias anuais de mortalidade e da incidência por sexo, faixa etária e formas clínicas da doença com base nas informações dos bancos de dados do Sistema de Informações de Tuberculose da Secretaria de Saúde do Estado da Bahia e do Sistema de Informações de Mortalidade do Ministério da Saúde. Analisou-se a distribuição espacial da tuberculose segundo distritos sani- tários. Ficou evidente o predomínio dos casos e óbitos no sexo masculino e na faixa etária de 15 a 39 anos. A forma pulmonar apresentou uma maior taxa de incidência e de mortalidade. Sobre a hipótese de que, a co-infecção AIDS/tuberculose possa contribuir para manter elevadas taxas de mortalidade, os dados existentes não corroboram para esta associação. A maior ocorrência de tuberculose em determinados distritos sanitários pode estar associada à densidade populacional e às condições desfavoráveis de vida.

Tuberculose; Sistemas de Informação; Mortalidade; Incidência 


\section{Colaboradores}

Ambos os autores participaram de todas as etapas de redação do artigo.

\section{Referências}

1. World Health Organization. Global tuberculosis control: report. Geneva: World Health Organization; 1998.

2. Dye C, Scheele S, Dolin PJ, Pathania V, Raviglione MC. Consensus statement. Global burden of tuberculosis: estimated incidence, prevalence, and mortality by country. WHO Global Surveillance and Monitoring Project. JAMA 1999; 282:677-86.

3. Superintendência de Estatística e Informações. Anuário estatístico. Salvador: Superintendência de Estatística e Informações; 2000.

4. Silva Jr. JB. Tuberculose: guia de vigilância epidemiológica. J Bras Pneumol 2004; 30 Suppl 1:S57S86.

5. Antunes JLF, Waldman EA. Tuberculosis in the twentieth century: time-series mortality in São Paulo, Brazil, 1900-97. Cad Saúde Pública 1999; 15: 463-76.

6. Phelan F, Teale C. Tuberculosis in older people: is it on the increase? Trends in notifications in Leeds from 1976 to 1996. Age Ageing 2000; 29:319-23.

7. Chaimowicz F. Age transition of tuberculosis incidence and mortality in Brazil. Rev Saúde Pública 2001; 35:81-7.

8. Galesi VMN. Mortalidade por tuberculose no $\mathrm{Mu}-$ nicípio de São Paulo: análise de uma década, 1986 a 1995 [Dissertação de Mestrado]. São Paulo: Universidade de São Paulo; 1999.

9. Cavalcante SC. Epidemiologia da tuberculose no município do Rio de Janeiro: revisão dos casos notificados, 1995-1997. Bol Pneumol Sanit 1998; 6:82-92.

10. National Institute of Allergy and Infections Diseases, Respiratory Infections. The Jordan report: accelerated development of vaccines. Bethesda: National Institute of Allergy and Infections Diseases, National Institutes of Health; 2000.

11. Pereira SM. Efetividade da 1a dose da vacina BCG contra tuberculose em crianças de Salvador, Bahia [Tese de Doutorado]. Salvador: Universidade Federal da Bahia; 2000.

12. Costa MC, Mota EL, Pinto LL. Efeito protetor da BCG na meningite tuberculosa. Bol Oficina Sanit Panam 1991; 110:26-32.

13. Dupon M, Texier-Maugein J, Leroy V, Court JS. Tuberculosis and HIV infection: a cohort study of incidence and susceptibility to antituberculous drugs, Bordeaux, 1985-1993. Groupe d'Epidemiologie Clinique du SIDA en Aquitaine. AIDS 1995; 9:577-83.
14. Borroto-Gutiérrez S, Armas-Pérez L, GonzálezOchoa E, Peláez-Sánchez O, Arteaga-Yero AL, Sentilhes A, et al. Distribución y tendencia de tuberculosis por grupos de edades y por municipios en ciudad de la Habana, Cuba, (1986-1998). Rev Esp Salud Pública 2000; 74:507-15.

15. Natal SR. Incidência das manifestações pulmonares das micoses no HRPS. J Pneumol 1986; 12 Suppl 14:23-7.

16. Natal SR, Gerhardt Filho G. Tuberculose nos menores de 15 anos. Pulmão 1991; 1:103-6.

17. Organización Panamericana de la Salud. Evaluación epidemiológica de la tuberculosis. Tendencias en algunos países de las Américas. Bol Epidemiol (Wash.) 1987; 8:1-5.

18. Gerhardt Filho G, Hijjar MA. Aspectos epidemiológicos da tuberculose no Brasil. J Pneumol 1993; 19:4-10.

19. I Consenso Brasileiro de Tuberculose 1997. J Pneumol 1997; 23:294-342.

20. Hijjar MA. Epidemiologia da tuberculose no Brasil. Inf Epidemiol SUS 1992; 6:53-81.

21. Kerr-Pontes LR, Oliveira FA, Freire CA. Tuberculose associada à AIDS, situação da Região do Nordeste brasileiro. Rev Saúde Pública 1997; 31:323-9.

22. Reinhard C, Paul WS, McAuley JB. Epidemiology of pediatric tuberculosis in Chicago, 1974 to 1994: a continuing public health problem. Am J Med Sci 1997; 313:336-40.

23. Mangtani P, Jolley DJ, Watson JM, Rodrigues LC. Socioeconomic deprivation and notification rates for tuberculosis in London during 1982-91. BMJ 1995; 310:963-6.

24. Carneiro NMB, Mota E. Tuberculose em Salvador, Bahia: incidência e algumas variáveis sócio-demográficas em 1980. Rev Baiana Saúde Pública 1986; 1/3:68-80.

25. Fundação Nacional de Saúde. Plano nacional de controle da tuberculose. Brasília: Ministério da Saúde; 1999

26. Gerhardt Filho G, Natal SR. Eficiência do tratamento da tuberculose no Brasil. Inf Epidemiol SUS 1995; 4:95-8.

Recebido em 22/Jul/2005

Versão final reapresentada em 19/Abr/2006

Aprovado em 23/Mai/2006 